\title{
Accidental Intramuscular Isoprenaline in Early Pregnancy: The Effects, Management and Outcome
}

\author{
M MOSTAFI $^{\mathrm{a}}$, MARAHMAN ${ }^{\mathrm{b}}$, MT MOLLICK $^{\mathrm{c}}$, MN HAQ $^{\mathrm{d}}, \mathrm{ARAFI}^{\mathrm{e}}$
}

\begin{abstract}
Summary:
Isoprenaline is a beta-adrenergic agonist, used for the treatment of cardiovascular emergencies like hypotensive shock or sever bradycardia. This drug is not indicated to use in bolus form either intramuscularly or intravenously. Accidentally a 22 year old pregnant woman was given an intramuscular injection of two
\end{abstract}

\section{Introduction:}

Isoprenaline is a synthetic sympathomimetic amine that is structurally related to epinephrine. It is used for the treatment of bradycardia, heart block, hypotensive conditions and rarely for asthma. It is a non-selective beta-adrenergic agonist, used as intravenous infusion, oral, intranasal or subcutaneous. Therapeutic dose of isoprenaline varies depending on indication of use. The plasma half-life for isoprenaline is approximately two hours. Usually it is given in a dose of IV infusion 5 ìg/ min initially, then doses of 2-20 ìg/min based on patient's response. The lethal dose of isoprenaline is $10-50 \mathrm{ig} / \mathrm{kg}$ body weight (in animal). Overdose of isoprenaline may cause tachycardia, palpitations, angina, Adams-Stokes attacks, pulmonary edema, hypertension, hypotension, ventricular arrhythmias, dyspnea, nervousness, headache, dizziness, nausea, visual blurring, flushing of the skin, sweating, tremor, weakness, and pallor. Cardio toxicity causing fatal outcome are ventricular tachycardia, ventricular fibrillation and cardiac asystole ${ }^{1}$.

a. Brig Gen Prof Mamun Mostafi, Head, Dept. of Medicine, Rangpur Medical College \& CMH.

b. Maj Md AnisurRahman, Combined Military Hospital (CMH), Rangpur Cantonment, Rangpur.

c. Lt Col Md Torab Mollick, Combined Military Hospital (CMH), Rangpur Cantonment, Rangpur.

d. Lt Col Md Naimul Haq, Combined Military Hospital (CMH), Rangpur Cantonment, Rangpur.

e. Lt Col Ahmed Rafi, Combined Military Hospital (CMH), Rangpur Cantonment, Rangpur.

Address of Correspondence: Brig Gen Prof Mamun Mostafi, Head, Department of Medicine, Rangpur Army Medical College and CMH Rangpur, E-mail: mamunmostafi@yahoo.com

Received: 19 October, 2014

Accepted: 7 February, 2016 mg Isoprenaline. It was a lethal dose, fortunately the patient survived. This article narrated the events, the ECG changes, management and outcome of the patient and pregnancy.

Key words: Isoprenaline, overdose, side effects, treatment.

(J Bangladesh Coll Phys Surg 2016; 34: 108-111)

However, lethal effects of isoprenaline toxicity can be minimized by propranolol infusion ${ }^{2}$.

\section{Case report:}

A 22 old woman, wife of a soldier of Bangladesh Army, was admitted to Combined Military Hospital (CMH), Rangpur through emergency and casualty department, with 14 weeks' history of amenorrhea, per vaginal bleeding and abdominal pain for three hours following sexual intercourse. She also had symptoms of early pregnancy like morning sickness. Clinically an average built lady weighing $48 \mathrm{~kg}$, anxious, pale, pulse 96 beats/ min, blood pressure 100/60 mm Hg, respiration 20 breaths/min, temperature $98^{0} \mathrm{~F}$. Abdominal examination showed mild lower abdominal tenderness and per vaginal bloody discharge. She was diagnosed as a case of threatened abortion and subsequently it was confirmed by urine analysis and ultrasound of pelvic organs. She was managed by physical rest, intravenous fluid, sedatives and injection progesterone (proluten depot) intramuscular weekly (total 10 doses). On third day of admission she was advised discharge from the hospital after first dose of weekly injection progesterone.

Instead of injection progesterone, injection isoprenaline one $\mathrm{ml}$ ( $2 \mathrm{mg}$, about $41 \mathrm{ig} / \mathrm{kg}$ weights) was given intramuscular mistakenly by the duty nurse. It was discovered by identifying the used ampoule of isoprenaline injection.

Immediately after the injection, patient developed palpitation, chest pain, breathlessness, profuse sweats, tremor, agitation, and restlessness. There was headache, dizziness, nausea, vomiting and visual blurring followed by drowsiness. Examination revealed cloudiness of consciousness, tachycardia with gallop; lungs were clear. Patient was immediately shifted to intensive care 
unit (ICU); her heart rate was 190 beats/min, blood pressure 40/30 mm Hg, respiration 40 breaths/min there was central cyanosis.

12 lead electrocardiogram (ECG) could not be done because of restlessness, however she was on continuous cardiac monitor and ECG tracings were available instantly. creatine phosphokinase (CPK), CK-MB and all other relevant investigations were conducted including random blood sugar, serum electrolytes, renal function and liver function test, all of them were normal.
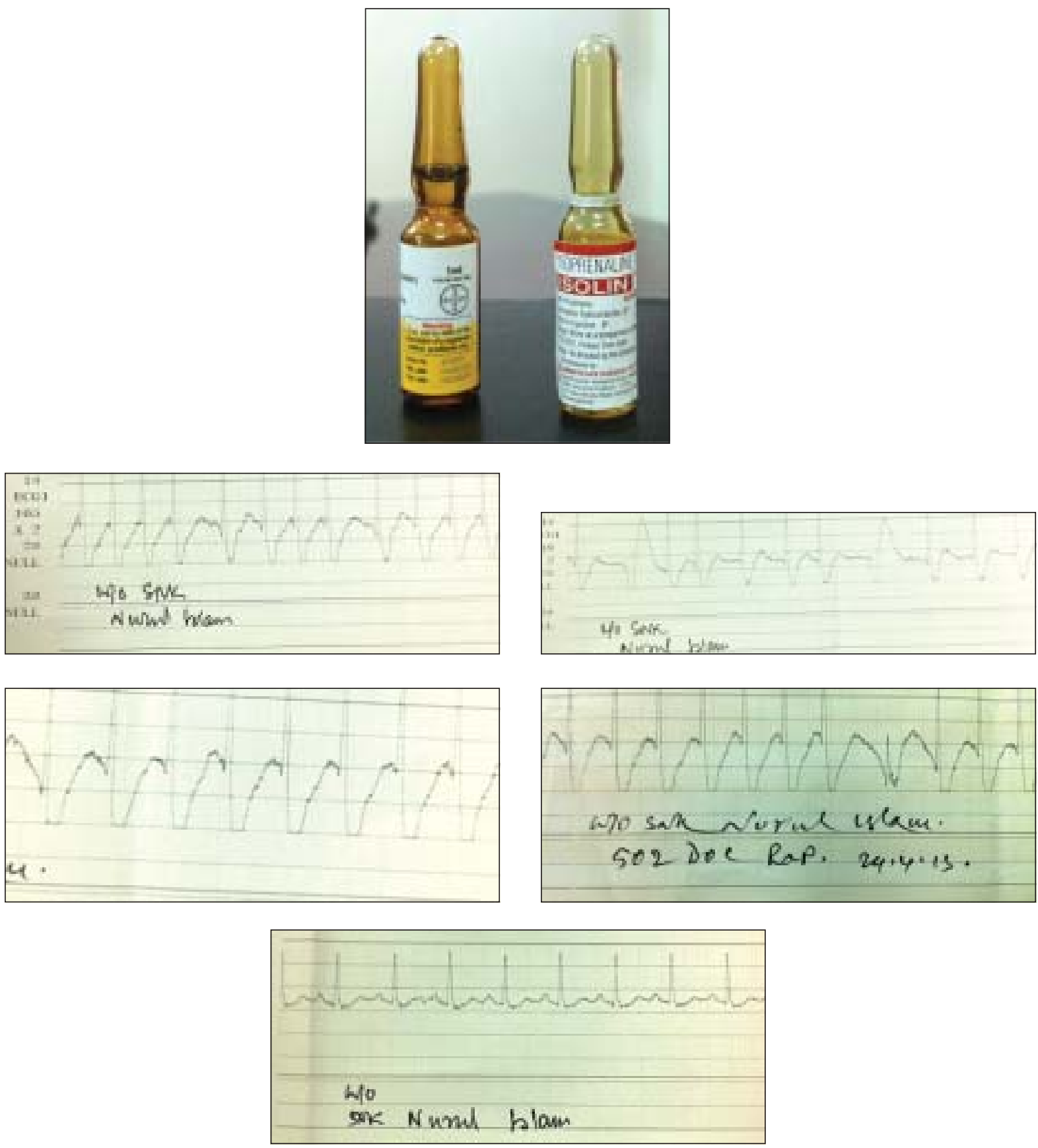

A- Isoprenaline and proluten depot injection vials; B, C, D, \& E- ECG changes after intramuscular isoprenaline; F - ECG tracing after 12 hours.

Fig. Isoprenaline injection and ECG changes 
She was advised intravenous propranolol, but could not be provided because of nonavailability in the local market. Oral Propranolol was started, $40 \mathrm{mg}$ stat and two hourly. She was also treated with carotid massage, intravenous fluid, sedatives and intravenous omeprazole along with high flow oxygen. She was on continuous cardiac monitoring and pulse oxymeter. Other parameters were followed up continuously minute to minute. Patient developed different types of ECG abnormalities (atrial tachycardias and VPC) as shown in the picture which finally returned to normal sinus rhythm.

After about four hours, patient's blood pressure improved (BP-70-90/30-50 mmHg), Pulse 130-100 beats/ min, patient felt better, consciousness regained and chest was clear. Patient gradually settled over 6-8 hours. There after patient was stable heamodynamically and on fifth day patient was discharged from hospital without any adverse outcome. Later on the patient had a normal vaginal delivery with a healthy male child.

\section{Discussion:}

Isoprenaline is a non-selective beta-adrenergic agonist, by activating $\hat{a}_{1}$-receptors on the heart, it induces positive chronotropic, dromotropic, and inotropic effects. Isoprenaline's effects on the cardiovascular system (non-selective) relate to its actions on cardiac $\hat{a}_{1}$ receptors and $\hat{a}_{2}$ receptors on smooth muscle within the tunica media of arterioles. It has positive inotropic and chronotropic effects on the heart. Beta ${ }_{2}$ adrenoceptor stimulation in arteriolar smooth muscle induces vasodilation. It's inotropic and chronotropic effects elevate systolic blood pressure, while its vasodilatory effects tend to lower diastolic blood pressure. The overall effect is to decrease mean arterial pressure due to the $\hat{a}_{2}$ receptor's vasodilation ${ }^{3}$.

Isoprenaline toxicity has a historical background. An epidemic of deaths within a group of individuals who were being treated for asthma was detected between 1963 and 1968 in Europe, and Australia. This was later found to be largely attributed to isoprenaline inhalers which were being used at five times the dose used in USA and Canada. Shortly after realizing overdose of the drug was causing many deaths, the medication was withdrawn and the number of asthmatics dying was quickly decreased ${ }^{4}$. The exact cause of this increased mortality was not known but it may be related to the excessive use of aerosols containing isoprenaline ${ }^{5}$. Analysis of individual cases has shown that patients have died suddenly and unexpectedly. Descriptions of their clinical state before death were understandably scanty but found a marked tachycardia in their eight patients; in one an electrocardiogram showed multiple ectopic beats ${ }^{6-7}$.

The acute toxicity of isoprenaline cause a striking drop in blood pressure, and repeated large doses may result in cardiac enlargement and focal myocarditis. Accidental overdosage of isoprenaline is evidenced mainly by tachycardia or other tachyarrhythmias, palpitations, angina, hypotension or hypertension ${ }^{1}$.

The effects of the intravenous injection of isoprenaline on heart rate and arterial pressure have been studied in animals. The fatal dose of isoprenaline (10-50 ìg/kg) reduced arterial and pulse pressures. Ventricular fibrillation did not occur; death occurred from cardiac asystole. Death was produced in a similar way in animals with hypoxaemia by giving four or five doses of isoprenaline $(2.5 \mathrm{ig} / \mathrm{kg})$ at five minutes intervals or by two doses of $25 \mathrm{ig} / \mathrm{kg}$. The final reduction in arterial pressure during a fatal response resulted from a reduction in cardiac contractility ${ }^{9}$.

In case of accidental toxicity of isoprenaline patient should be closely observed with cardiac rhythm monitoring by ECG, monitoring of pulse, blood pressure, respiration, consciousness and arterial oxygen saturation by pulse oxymetry. The lethal effects of isoprenaline could be prevented by intravenous propranolol. The cardiac toxic effects of isoprenaline may be potentiated by hypoxia. Intensive oxygen therapy is also required. It is not known whether isoprenaline is dialyzable or not ${ }^{9}$.

In the reported patient the administered dose was very high; about 41 ig/kg, which is a lethal dose theoretically. It could be easier if injectable propranolol was available. In spite of that the success was possibly because of young otherwise healthy heart, immediate attention and subsequent management.

Extensive search in internet showed no case report relevant to accidental parental isoprenaline toxicity. To our best knowledge it is the first published case report of such toxicity in Bangladesh and probably in the world. 


\section{Conclusion:}

Accidental drug overdose or toxicity is not uncommon in the medical institutions; human error is always there. A confession of the mistake, detecting the used drugs and an immediate appropriate intervention can save the life in this dangerous and precarious situation.

\section{References:}

1. Current medication information; Isoproterenol Hydrochloride; The Daily Med; The US National Library of Medicine; 'http://dailymed.nlm.nih.gov/dailymed; Revised: 3/2013

2. Shanks, R.G. The effect of Propranolol, the cardiovascular responses to isoprenaline, adrenaline and nor-adrenaline in the anaesthetized dog. Brit J. Pharmaco 26:322:1966

3. B. G. Katzung; Adrenoceptor activating \& other sympathomimetic Drugs; Katzung Pharmacology, $9^{\text {th }}$ edition; Section II, Chapter 9; page 169- 199.
4. Pierce, Neil and Hensley, Michael J. "Epidemiologic Studies of Beta Agonists and Asthma Deaths". Epidemiologic Studies 20 (2):1998.

5. Speizer. E.E, Heaf. P, Doll, R and Strange L,B. Observation on recent increase in mortality from asthma; Brith med journal 1, 335-39; 30 October,1968

6. Greenberg MJ, Pines A. Pressurized Aerosols in Asthma. Br Med J. 1967 Mar 04;1(5539):a563-563

7. Investigation into use of drugs preceding death from asthma, British med journal 1, 339-43, 30 October, 1968.

8. Harris, Wallard, Clyde D, Schoenfeld, Arnold M. Weisseren: Effects of adrenergic receptor activation and blockade on the systolic pre-ejection period, heart rate and arterial pressure in man: The jourl of clini. invest, vol- 46, no- 11, 1967.

9. J. M. Collins, D. G. Mc Devitt, R. G. Shanks, and J. G. Swanton; The cardio-toxicity of isoprenaline during hypoxia; British Journal of Pharmacology Volume 36, Issue 1, published online: 19 July 2012. 